\title{
Berichtigungen.
}

S. 291 Zeile 7 v. o. V. s. statt V. c.

" 295 " 4 v. u. C. a. " c. a.

" 297 " 13 v. o. (Fig. 6, 10, 11, Si u. Si*) zu tilgen und Z. 15 hinter ,angefügt" zu setzen.

"301 " 9 v. o. 23 A statt 23.

„306 " 1 v o. Fig. 23 \& statt Fig. 23.

"306 " 25 v. o. 27 Ps. statt 25.

„315 " 14 v. o. zu tilgen (Fig. 25.)

" 321 " 23 v. o. Fig. 23 statt Fig. 24.

, 331 , 16 v. o. V. v. s. statt V. v.

\section{Ueber Molluskenaugen.}

Von

Justus Carriere.

Hierzu Tafel XXIII.

Eine kürzlich ausgegebene Untersuchung von Rawitz ${ }^{1}$ ) gibt mir willkommene Gelegenheit, anf die im Jahre 1886 in Band VI, Heft 4 der Mittheilungen der zoologischen Station in Neapel veröffentlichte Abhandlung W. Pattens zurückzukommen. Sie war betitelt: „Eyes of Molluses and Arthropods", und auf der zoologischen Station in Neapel mit den neuesten Methoden und Instrumenten ausgefuhrt, ein umfangreiches Werk. Mir aber war es kein Vergnugen und ich bedauerte die wenigen Leidensgefährten, die gleich mir gezwungen waren sich durch die Fülle von Neuheiten durchzuarbeiten, die dasselbe in seinen beiden Theilen, dem beschreibenden und dem hypothetischen, bot; denn obschon

1) Dr. Bernhard Rawitz. Der Mantelrand der Acephalen. Erster Theil. Ostracea. Jena 1888. Zeitschrift f. Naturwissenschaft XXII, N. F. XV. 